\title{
Rad51 inhibition sensitizes breast cancer stem cells to PARP inhibitor in triple-negative breast cancer
}

\author{
Dong Wang ${ }^{1}$, Ruikai Du and Suling Liu ${ }^{2,3^{*}}$
}

Breast cancer susceptibility gene 1 (BRCA1) is a tumor suppressor gene, and its protein BRCA1 plays a role in DNA repair [1]. BRCA1 is generally expressed in the cells of mammary glands and other tissues, helping to repair damaged DNA or disrupting cells when DNA cannot be repaired. When $B R C A 1$ is mutated and cannot function and therefore the damaged DNA cannot be repaired on time, the risk of breast cancer will greatly increase [2]. In BRCA1-mutant tumors, the capability of DNA damage repair is decreased, which makes tumor cells sensitive to DNA-damaging drugs; however, high BRCA1 activity weakens the effect of these drugs [3].

Poly(ADP-ribose) polymerase (PARP) is an enzyme that detects DNA single-strand breaks and mediates DNA repair. The inhibition of PARP leads to the accumulation of DNA fragmentation, especially in patients with loss of BRCA activity, and results in cells being killed by excessive DNA damage [4]. Preclinical models show that PARP inhibition selectively targets breast cancer cells lacking functional BRCA1 [5]. Accumulating evidence shows that cancer stem cells (CSCs) are responsible for the resistance to chemotherapeutic agents [6], but it is unknown whether targeted therapy (including PARP inhibition) has the same effect. As reported in the paper entitled "RAD51 mediates resistance of cancer stem cells to PARP inhibition in triple-negative breast cancer" [7] in a recent issue of Clinical Cancer Research, we found that PARP inhibitor (PARPi) only effectively targets BRCA1mutant bulk tumor cells and RAD51, a gene involved in DNA double-strand break repair [8], mediates this process, but BRCA1-wild-type cancer cells and BRCA1mutant CSCs are resistant to PARP inhibition. RAD51

\footnotetext{
*Correspondence: suling@fudan.edu.cn

${ }^{2}$ Key Laboratory of Breast Cancer in Shanghai, Cancer Institute,

Department of Breast Surgery, Fudan University Shanghai Cancer Center,

Shanghai 200032, P. R. China

Full list of author information is available at the end of the article
}

knockdown (KD) sensitizes both BRCA1-mutant CSCs and BRCA1-wild-type cells to PARP inhibition. It suggests an effective therapeutic intervention for targeting triple-negative breast cancer (TNBC).

In this study, we tested the cytotoxic effect of olaparib (a PARPi) on four TNBC cell lines including both BRCA1-mutant and BRCA1-wild-type cell lines: SUM149, SUM159, HCC1937, and MDA-MB-231. As expected, PARPi treatment resulted in fewer viable cells in BRCA1-mutant cell lines (SUM149 and HCC1937) compared with those with wild-type BRCA1 (SUM159 and MDAMB231). However, after 7 days of PARPi treatment in SUM149 and HCC1937 cells, the ALDEFLUORpositive cells (i.e., CSCs) [9] was enriched, indicating that these cells were not affected by PARP inhibition. We thus performed an exploratory polymerase chain reaction array of 84 key DNA repair genes and found that RAD51 has higher expression in CSCs than in bulk tumor cells in SUM149 cells. We infected SUM149 and SUM159 cells with a doxycycline-inducible $R A D 51$ short-hairpin RNA lentiviral system. Although not affecting CSC populations in SUM159 cells, RAD51 KD sensitized CSCs to PARPi in SUM149 cells. These results support our hypothesis that RAD51 mediates resistance of CSCs to PARPi in BRCA1mutant and BRCA1-wild-type breast cancer cells.

We next determined the effects of PARP inhibition and $R A D 51 \mathrm{KD}$ in an in vivo model of TNBC. The mice were divided into four treatment groups: vehicle control, olaparib, RAD51 KD (doxycycline water), and combined (olaparib plus RAD51 KD). For the mice injected with BRCA1-mutant SUM149 cells, RAD51 KD and PARP inhibition significantly inhibited tumor growth in the early stage. However, when treatment was given after the tumor grew to a certain stage ( $2 \mathrm{~mm}$ in diameter), PARP inhibition was not effective, whereas the effect of RAD51 KD was still significant. The combination of RAD51 KD and PARPi led to a more robust inhibition 
of tumor growth. To assess the effects of PARP inhibition on the CSC frequency, serial dilutions of SUM149 cells (control and treated) were re-injected into mouse mammary fat pads [10]. Compared with vehicle control, RAD51 KD alone and combined treatments reduced the CSC frequency by $70 \%$ and $90 \%$, respectively. There is a consistent result in BRCA1-wild-type SUM159-injected mice. Together, these results indicate that our therapeutic regimen targets both BRCA1-mutant CSCs and BRCA1wild-type cells (both CSC and bulk tumor populations), supporting our in vitro findings of the involvement of RAD51 in resistance to olaparib and, importantly, suggesting an effective therapeutic intervention for targeting TNBC.

In conclusion, we used in vitro models and mouse xenografts to demonstrate the importance of RAD51 in reversing PARPi resistance of CSCs in BRCA1-deficient and $B R C A 1$-wild-type TNBC in this study. Our findings suggest that resistance to PARPi may be overcome by targeting both CSCs and bulk tumor cells. To elucidate the clinical implications, we will further use the breast cancer patient-derived xenograft model to validate our current findings. Furthermore, by targeting RAD51, it may be possible to greatly expand the sensitivity of TNBC to PARPi, beyond those with defective BRCA1 proteins. This novel approach holds potentials for significantly improved therapies for TNBC.

\section{Authors' contributions}

DW, RD, and SL drafted and critically revised the manuscript. All authors read and approved the final manuscript.

\section{Author details}

${ }^{1}$ The CAS Key Laboratory of Innate Immunity and Chronic Disease, Hefei National Laboratory for Physical Sciences at the Microscale, School of Life Science and Medical Center, University of Science \& Technology of China, Hefei 230027, Anhui, P. R. China. ${ }^{2}$ Key Laboratory of Breast Cancer in Shanghai, Cancer Institute, Department of Breast Surgery, Fudan University Shanghai Cancer Center, Shanghai 200032, P. R. China. ${ }^{3}$ Institutes of Biomedical Sciences, Fudan University, Shanghai 200032, P. R. China.

\section{Acknowledgements}

This work was supported by National Natural Science Foundation of China grants (Nos. 81530075 and 81472741), the National Key Research and Development Program of China (Stem Cell and Translational Research 2016YFA0101202), and the Ministry of Science and Technology (MOST) grant (No. 2015CB553800).

\section{Competing interests}

The authors declare that they have no competing interests.

Received: 1 March 2017 Accepted: 27 March 2017

Published online: 30 March 2017

References

1. Venkitaraman AR. Cancer susceptibility and the functions of BRCA1 and BRCA2. Cell. 2002;108(2):171-82.

2. Friedenson B. The BRCA1/2 pathway prevents hematologic cancers in addition to breast and ovarian cancers. BMC Cancer. 2007:7:152.

3. Papadaki $C$, et al. ERCC1 and BRAC1 mRNA expression levels in the primary tumor could predict the effectiveness of the second-line cisplatinbased chemotherapy in pretreated patients with metastatic non-small cell lung cancer. J Thorac Oncol. 2012;7(4):663-71.

4. Chen A. PARP inhibitors: its role in treatment of cancer. Chin J Cancer. 2011;30(7):463-71.

5. Audeh MW, et al. Oral poly(ADP-ribose) polymerase inhibitor olaparib in patients with BRCA1 or BRCA2 mutations and recurrent ovarian cancer: a proof-of-concept trial. Lancet. 2010;376(9737):245-51.

6. Charafe-Jauffret $E$, et al. Breast cancer cell lines contain functional cancer stem cells with metastatic capacity and a distinct molecular signature. Cancer Res. 2009;69(4):1302-13.

7. Liu Y, et al. RAD51 mediates resistance of cancer stem cells to PARP inhibition in triple-negative breast cancer. Clin Cancer Res. 2017;23(2):514-22.

8. Galkin VE, et al. The Rad51/RadA N-terminal domain activates nucleoprotein filament ATPase activity. Structure. 2006;14(6):983-92.

9. Ginestier $\mathrm{C}$, et al. ALDH1 is a marker of normal and malignant human mammary stem cells and a predictor of poor clinical outcome. Cell Stem Cell. 2007;1(5):555-67.

10. Hu Y, Smyth GK. ELDA: extreme limiting dilution analysis for comparing depleted and enriched populations in stem cell and other assays. J Immunol Methods. 2009:347(1-2):70-8. 